\title{
Torsional oscillations of sunspots: magnetic and velocity fields observations
}

\author{
O. S. Gopasyuk \\ Crimean astrophysical observatory, Nauchny, Crimea, 98409, Ukraine \\ email: olg@crao.crimea.ua
}

\begin{abstract}
Torsional oscillations of sunspots were investigated in a penumbra on the observations in the photosphere of the longitudinal magnetic and velocity fields.
\end{abstract}

\section{Introduction}

Torsional oscillations of sunspots with the period about 6 days have been found by Gopasyuk (1981) on the basis of line-of-sight velocities in the photosphere, photoheliograms and $H_{\alpha}$ images of active regions.

These oscillations were used for investigations of magnetic field structures in the subphotospheric layers (Gopasyuk 1984). Torsional oscillations of sunspots are not unique, but rather an intrinsic feature of sunspot states (Gopasyuk \& Lyamova 1987).

The rotation of sunspots leads to a twisting of the lines of force their magnetic fields. An appearance of flares closely correlates with sunspot rotations (Gopasyuk 1965; Gopasyuk \& Lazareva 1986).

The sunspot rotations about the axis and torsional oscillations of sunspots were studied recently by Brown et al. (2003) on white-light images. In addition to the main observational data they included $\mathrm{H} \alpha$ and UV (1600 $\AA$, $171 \AA$ ) images and SOHO/MDI magnetograms.

Now we carried out the investigations of the torsional oscillations of single sunspot penumbrae using line-of-sight components of magnetic and velocity fields measured in the photospheric Fe I $\lambda 5253 \stackrel{A}{\text { line. }}$

\section{Observational date and results}

The observational data of single sunspots were taken in the Crimean astrophysical observatory with the double magnetograph on September 1978 and on August 2000 from the high-speed digital magnetograph of Solar Tower Telescope.

An axial symmetry of magnetic and velocity fields in single sunspots allows us to reconstruct all three components of both vectors from their line-of-sight components (Kinman 1952; Gopasyuk \& Gopasyuk 1998).

For each of components of magnetic and velocity fields sinusoid

$$
y=A \sin \left(\frac{2 \pi t}{T}+\alpha\right)
$$

was fitted. $A, T$ and $\alpha$ are the amplitude, period and initial phase of oscillations, respectively. The results of fitting are shown in figure 1 .

The data of calculations showed, that the period of oscillations in the penumbra of investigated four sunspots was within the interval $3.4-7.7$ days. The period for all components of both fields of one and the same sunspot was identical. The phase difference 

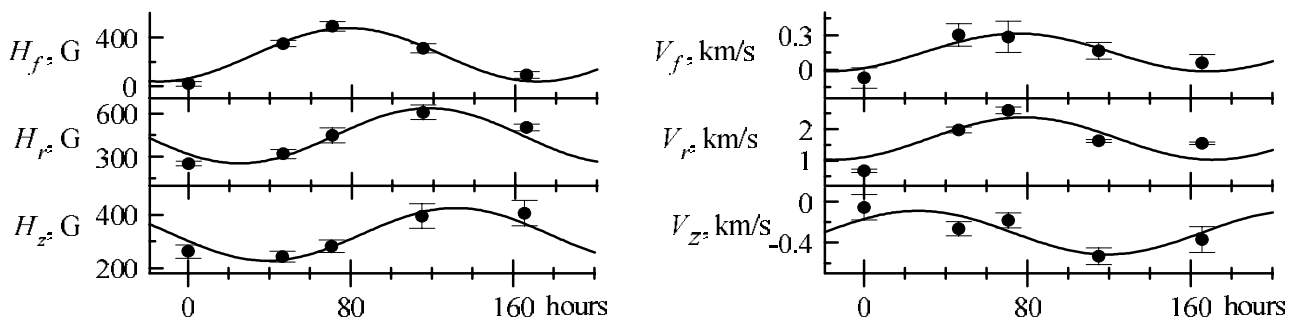

Figure 1. Changes with time of the magnetic and velocity field components in the sunspot on $13-20$ September, 1978. The period of oscillations is 7.7 days.

between oscillations of the azimuthal velocity and all other components of the velocity and magnetic fields is in the interval $0-\pi$, except four cases that exceed this value. Oscillations of the azimuthal velocity are ahead on the phase oscillations of components of both velocity and magnetic fields.

The initial phases of oscillations of all components (the magnetic and velocity fields) increase with latitude of a sunspot.

If the mass density in a penumbra is taken $10^{-4} \mathrm{~kg} / \mathrm{m}^{3}$ (the same as in the photosphere) the kinetic energy is less than the magnetic energy for the same components. In most cases this ratio is within the interval $0.1-0.01$.

\section{Conclusions}

Our observational data showed that the azimuthal velocity calculated from the velocity field is more than the azimuthal speed measured from photoheliograms. It confirms more early results derived by Gopasyuk (1981). At the same time twisting of the lines of force of the magnetic field on the external penumbra boundary corresponds to the angle of the sunspot turn calculated from photogeliograms (Gopasyuk 1987). It may be these effects are connected with an ununiformity of magnetic fields and plasma.

The period of oscillations in the penumbra of sunspots was within the interval $3.4-$ 7.7 days. The period for all components of both fields of one and the same sunspot was identical. The phase of the azimuthal component of the velocity is ahead of the phases of all other components.

If the density of plasma in a penumbra is $10^{-4} \mathrm{~kg} / \mathrm{m}^{3}$ (the same as in the photosphere) the magnetic energy of oscillations exceeds of the kinetic energy of the same components by a factor of $10-100$.

We concluded that these oscillations are forced oscillations.

\section{References}

Brown, B.N., Nightingale, R.W., Alexander, D. et al. 2003 Solar Phys. 216, 79-108.

Gopasyuk, S.I. 1965 Izv. Krymsk. Astrofiz. Obs. 33, 100-110.

Gopasyuk, S.I. 1981 Izv. Krymsk. Astrofiz. Obs. 64, 108-118.

Gopasyuk, S.I. 1984 Astron. Zh. 61, 157-162.

Gopasyuk, S.I. 1987 In Proc. of 10th European regional astronomy meeting of the IAU (ed. Hejna, L. \& Sobotka, M.), pp. 137-145.

Gopasyuk, S.I. \& Gopasyuk, O.S. 1998 Kinematics and Physics of celestial bodies 14, 389-400.

Gopasyuk, S.I. \& Lazareva, L.F. 1986 Izv. Krymsk. Astrofiz. Obs. 74, 84-92.

Gopasyuk, S.I. \& Lyamova, G.V. 1987 Izv. Krymsk. Astrofiz. Obs. 77, 17-24.

Kinman, T.D. 1952 Mon. Not. R. Astr. Soc. 112, 425-444. 